\section{Identificación de microorganismos asociados con rinosinusitis crónica en pacientes adultos con inmunodeficiencia común variable}

Gabriela Angulo-Pérez ${ }^{1}$, Eulalio Vivar-Acevedo ${ }^{1}$, Diana Andrea Herrera-Sánchez ${ }^{2}$

Resumen

ANTECEDENTES: la prevalencia de rinosinusitis crónica en pacientes adultos con inmunodeficiencia común variable (IDCV) es de $52 \%$. Los pacientes con esta enfermedad tienen mayor frecuencia de rinosinusitis crónica, enfermedad inflamatoria que afecta a la mucosa de uno o más senos paranasales y la cavidad nasal.

OBJETIVO: identificar los microorganismos de secreción del meato medio obtenida por endoscopia asociados con rinosinusitis crónica en pacientes adultos con inmunodeficiencia común variable (IDCV).

MATERIAL Y MÉTODO: estudio descriptivo, transversal, que incluyó a pacientes adultos con inmunodeficiencia común variable, de quienes se obtuvo una muestra vía endoscópica de secreción del meato medio de ambas fosas nasales, que se envió a cultivo para bacterias aerobias, anaerobias y hongos. Se obtuvo consentimiento informado de todos los pacientes.

RESULTADOS: se estudiaron 29 pacientes: 18 mujeres y 11 hombres, con edad promedio de $40 \pm 13$ años. Los resultados obtenidos fueron: 2 muestras de pacientes no tuvieron desarrollo microbiano, 24 tuvieron desarrollo de bacterias aerobias, en 3 casos hubo crecimiento fúngico sin desarrollo de bacterias anaerobias.

CONCLUSIONES: nuestros resultados muestran que los microorganismos asociados con rinosinusitis crónica en pacientes adultos con inmunodeficiencia común variable más comunes son: Moraxella catarrhalis, Staphylococcus, Sphingomonas paucimobilis y Citrobacter koseri; los agentes micóticos asociados fueron: Candida albicans y Aspergillus fumigatus.

PALABRAS CLAVE: microorganismos, rinosinusitis crónica, inmunodeficiencia común variable, meato medio, endoscópico.

Rev Alerg Méx 2016 Jan-Mar;63(1):26-31.

\section{Identification of microorganisms related to chronic rhinosinusitis in adult patients with variable common immunodeficiency.}

Gabriela Angulo-Pérez ${ }^{1}$, Eulalio Vivar-Acevedo ${ }^{1}$, Diana Andrea Herrera-Sánchez ${ }^{2}$

\section{Abstract}

BACKGROUND: The prevalence of chronic rhinosinusitis in adult patients with common variable immunodeficiency (CVID) is $52 \%$. The
${ }^{1}$ Servicio de Otorrinolaringología y Cirugía de Cabeza y Cuello.

${ }^{2}$ Servicio de Alergia e Inmunología Clínica.

Hospital de Especialidades, Centro Médico Nacional Siglo XXI, Instituto Mexicano del Seguro Social, Ciudad de México.

Recibido: 13 de octubre 2015

Aceptado: 8 de diciembre 2015

Correspondencia

Dra. Gabriela Angulo Pérez

apybag@hotmail.com

Este artículo debe citarse como Angulo-Pérez G, Vivar-Acevedo E, Herrera-Sánchez DA. Identificación de microorganismos asociados con rinosinusitis crónica en pacientes adultos con inmunodeficiencia común variable. Rev Alerg Méx 2016 ene-mar;63(1):26-31. 
patients with CVID show higher incidence of chronic rhinosinusitis, which is an inflammatory disease that affects the lining of one or more paranasal sinuses and nasal cavity.

OBJECTIVE: To identify the microorganisms in the middle meatus secretion obtained by endoscopy associated with chronic rhinosinusitis in adult patients with common variable immunodeficiency (CVID).

MATERIAL AND METHOD: A descriptive, cross-sectional study, which included adult patients with CVID, from whom a sample endoscopic middle meatus secretion from both nostrils was obtained and sent to culture for aerobic, anaerobic bacteria and fungi. Informed consent of all patients was obtained.

RESULTS: 29 patients were studied: 18 women and 11 men with a mean age of $40 \pm 13$ years. The results were: 2 samples showed no microbial growth, 24 showed growth of aerobic bacteria, 3 cases had fungal growth without development of anaerobic bacteria.

CONCLUSIONS: Our results show that the most common microorganisms associated with CSR in adult patients are: Moraxella catarrhalis, Staphylococcus, Sphingomonas paucimobilis and Citrobacter koseri, and associated fungal agents were: Candida albicans and Aspergillus fumigatus.

KEYWORDS: microorganisms; chronic rhinosinusitis; common variable immunodeficiency

\author{
${ }^{1}$ Servicio de Otorrinolaringología y Cirugía \\ de Cabeza y Cuello. \\ 2 Servicio de Alergia e Inmunología Clínica. \\ Hospital de Especialidades, Centro Médico \\ Nacional Siglo XXI, Instituto Mexicano del \\ Seguro Social, Ciudad de México. \\ Correspondence \\ Dra. Gabriela Angulo Pérez \\ apybag@hotmail.com
}

\section{ANTECEDENTES}

La rinosinusitis crónica es una enfermedad inflamatoria que afecta a la mucosa de uno o más senos paranasales y la cavidad nasal; en pacientes con inmunodeficiencia común variable su prevalencia es de $52 \%{ }^{1}$

A pesar de su prevalencia, no se han establecido los mecanismos patogénicos y los agentes implicados en esta enfermedad. No está claro cuáles son los gérmenes responsables en la sobreproducción de eosinófilos y linfocitos que ocurre en la rinosinusitis crónica que perpetúa la respuesta inflamatoria de la mucosa nasosinusal. ${ }^{2,3}$

Los estudios de microbiología en rinosinusitis crónica realizados en pacientes adultos con inmunodeficiencia común variable muestran variabilidad en sus resultados debido al uso de diferentes técnicas para la obtención de las muestras, administración repetida de antibióticos y la dificultad para reconocer si los microorganismos aislados son patógenos verdaderos o agentes colonizadores. ${ }^{2,3}$

La endoscopia nasal es una técnica que permite obtener la muestra directamente del sitio de drenaje de los senos paranasales, con mínima incomodidad para el paciente, lo que permite determinar la microbiología de los senos paranasales con baja probabilidad de contaminación. ${ }^{4-6}$

Araujo y su grupo validaron el uso de la endoscopia nasal para la obtención de secreción del meato medio y determinar de esta manera los microorganismos en pacientes con rinosinusitis crónica. $^{4}$ 
Jiang y colaboradores demostraron que el meato medio es el lugar más adecuado para la toma de muestras, es el lugar de drenaje del seno maxilar, las celdillas etmoidales anteriores y el seno frontal; los microorganismos de esta área reflejan mejor la microbiología de los senos paranasales en comparación con otras técnicas: exudado nasal y punción del antro maxilar. Establecieron el uso de aspiración de meato medio como el mejor método para identificar los microorganismos en pacientes con diagnóstico de rinosinusitis crónica. ${ }^{7,8}$

Araujo y colaboradores demostraron que $80 \%$ de las muestras obtenidas por punción del seno maxilar y las obtenidas por secreción de meato medio tenían crecimiento del mismo microorganismo. Este estudio sugiere que el cultivo obtenido guiado por endoscopia del meato medio es una alternativa viable y eficiente en relación con la punción del antro maxilar, tiene las ventajas de ser un método no invasivo y menos costoso. ${ }^{4}$

No hay estudios consistentes que definan el tipo de las bacterias causales de rinosinusitis crónica en pacientes adultos con inmunodeficiencia común variable. Por esta razón decidimos estudiar la incidencia de microorganismos presentes en la secreción de meato medio obtenida vía endoscópica en pacientes con rinosinusitis crónica e inmunodeficiencia común variable. La obtención de estas muestras es importante para guiar el tratamiento adecuado de acuerdo con el cultivo y la sensibilidad a los antimicrobianos.

\section{MATERIAL Y MÉTODO}

Estudio descriptivo, transversal, que incluyó a 29 pacientes, 18 mujeres y 19 hombres con límites de edad de 18 a 69 años.

Los criterios de inclusión fueron: pacientes adultos con diagnóstico de inmunodeficiencia común variable (criterios de la Sociedad Europea de Inmunodeficiencias Primarias), pertenecientes a la Clínica de Inmunodeficiencias del Hospital de Especialidades Dr. Bernardo Sepúlveda. Se excluyeron los pacientes que habían consumido antimicrobianos 20 días antes de la obtención de la muestra.

Se obtuvo la muestra con la siguiente técnica: se colocó algodón con anestesia y vasoconstrictor local lidocaína 1\% con epinefrina $0.25 \%$ durante 10 minutos, se retiraron los algodones, se obtuvo con hisopo secreción del meato medio, dirigido con ayuda de endoscopio Storz de $0^{\circ}$ dirigido hacia el meato medio. Se transportó la muestra en medio Stuart, se Ilevó al laboratorio de nuestro hospital en un tiempo promedio menor a 2 horas y las analizó el mismo experto.

En el laboratorio las muestras se inocularon para bacterias aerobias en medio MacConkey (peptonas, sales biliares, cristales púrpura, $\mathrm{pH}$ neutral) y agar sangre (agar Columbia $+5 \%$ de sangre de cordero), incubados a $37^{\circ} \mathrm{C}$ durante 48 horas. El mismo experto identificó los microorganismos.

La búsqueda de bacterias anaerobias se realizó cultivando la secreción obtenida en medio agar chocolate (agar, peptona carne, tripteína, extracto de levadura, extracto de corazón, almidón soluble, cloruro de sodio, sangre ovina, suplemento, agua purificada), en condiciones anaerobias a $35^{\circ} \mathrm{C}$ durante cinco días y revisadas cada 24 horas.

Para el análisis fúngico, las muestras se cultivaron en medio Sabouraud (peptona, tripteína, glucosa, cloranfenicol y agar). Los especímenes se cultivaron a $35^{\circ}$. Los cultivos se revisaron cada 24 horas durante tres días.

El mismo experto evaluó todas las muestras. 
Este estudio lo aprobó el Comité de Ética local. Los pacientes firmaron un consentimiento informado y se les dieron a conocer los resultados.

\section{RESULTADOS}

De los 29 pacientes estudiados (18 mujeres y 11 hombres, con edad promedio de $40 \pm 13$ años), dos muestras no mostraron desarrollo antimicrobiano, 24 tuvieron desarrollo de bacterias aerobias, en tres casos hubo crecimiento fúngico y no se encontró crecimiento de bacterias anaerobias.

Las bacterias aerobias encontradas fueron grampositivas en 18 casos y gramnegativas en nueve.

Las bacterias grampositivas que mostraron mayor desarrollo fueron: Staphylococcus epidermidis en 10 muestras, Staphylococcus aureus en 4 muestras, Staphylococcus haemolyticus en una muestra.

Las bacterias gramnegativas encontradas fueron: Moraxella catarrhalis en seis muestras, dos muestras con Sphingomonas paucimobilis y una muestra con crecimiento de Citrobacter koseri.

Crecimiento fúngico: Candida albicans tuvo crecimiento en dos muestras $(8 \%$ del total de muestras obtenidas) y Aspergillus fumigatus en una muestra (4\%). Cuadro 1

\section{DISCUSIÓN}

En años recientes se han realizado diversos estudios para identificar microorganismos de secreción del meato medio guiado por endoscopia, ${ }^{2-5}$ pero hay poca información en pacientes adultos con inmunodeficiencia común variable (Cuadro 2).

En el estudio de microorganismos asociados con rinosinusitis crónica debe considerarse el
Cuadro 1. Porcentaje de bacterias aerobias encontradas en secreción del meato medio de los 29 pacientes

\begin{tabular}{lc}
\hline Bacterias & Núm. (\%) \\
Grampositivas & $18(67)$ \\
Staphylococcus epidermidis & $10(42)$ \\
Staphylococcus aureus & $4(17)$ \\
Staphylococcus haemolyticus & $1(4)$ \\
Gramnegativas & $9(33)$ \\
Moraxella catarrhalis & $6(25)$ \\
Sphingomonas paucimobilis & $2(8)$ \\
Citrobacter koseri & $1(4)$ \\
\end{tabular}

método de obtención de la muestra, el medio de transporte y el tiempo en que se analizó la muestra. En nuestro estudio la muestra se obtuvo de secreción del meato medio por vía endoscópica y la muestra se entregó en menos de una hora posterior a la recolección.

Las bacterias aerobias gramnegativas aisladas en nuestro estudio fueron: Moraxella catarrhalis $(n=6,25 \%)$, Sphingomonas paucimobilis $(n=2$, $8 \%$ ) y Citrobacter koseri ( $n=1,4 \%)$; de los estudios revisados en ninguno se aisló Sphingomonas paucimobilis.

Sphingomonas paucimobilis es un bacilo gramnegativo que se ha aislado en pacientes con bacteremia, meningitis, peritonitis, infecciones cutáneas, abscesos viscerales, pero existe escasa bibliografía acerca de este microorganismo asociado con rinosinusitis crónica en pacientes con inmunodeficiencia común variable. ${ }^{9}$

Citrobacter koseri se aisló en nuestro estudio en una muestra (4\%) y sólo en el estudio de Panduranga $^{5}$ se aisló Citrobacter koseri en $1 \%$ de los casos. En el estudio de Daza se incluyeron 25 pacientes con rinitis crónica y este microorganismo se identificó como patógeno causal de rinitis crónica en cuatro pacientes. ${ }^{10}$

Este estudio encontró como microorganismos asociados con rinosinusitis crónica más 
Cuadro 2. Análisis comparativo de flora microbiana obtenida por vía endoscópica en pacientes sin inmunodeficiencia común variable $\mathrm{e}^{2,4,5}$

\begin{tabular}{lcccc}
\hline Patógenos & $\begin{array}{c}\text { Panduranga, 2013 } \\
(\mathbf{n = 1 0 0})\end{array}$ & $\begin{array}{c}\text { Mantovani, 2010 } \\
(\mathbf{n = 6 2 )}\end{array}$ & $\begin{array}{c}\text { Araujo, 2007 } \\
(\mathbf{n = 1 3 4 )}\end{array}$ & $\begin{array}{c}\text { Angulo, 2015 } \\
(\mathbf{n = 2 9 )}\end{array}$ \\
Staphylococcus aureus & 43 & 4 & 53 & 4 \\
Staphylococcus epidermidis & 0 & 4 & 19 & 10 \\
Staphylococcus haemolyticus & 0 & 0 & 2 & 1 \\
Streptococcus pneumoniae & 1 & 3 & 22 & 0 \\
Pseudomonas aeruginosa & 1 & 8 & 10 & 0 \\
Klebsiella pneumoniae & 9 & 1 & 0 & 0 \\
Enterobacter sp & 1 & 2 & 3 & 1 \\
Citrobacter koseri & 1 & 0 & 0 & 1 \\
Sphingomonas paucimirabilis & 0 & 0 & 0 & 2 \\
Candida albicans & 3 & 0 & 6 & 1 \\
Aspergillus fumigatus & 4 & 0 & 1 &
\end{tabular}

frecuentes a Moraxella catarrhalis (25\%) y Staphylococcus aureus (17\%). En el estudio de Mantovani, efectuado en 62 pacientes con rinosinusitis crónica sin inmunodeficiencia común variable (2010), ${ }^{2}$ los microorganismos aislados más comunes fueron Pseudomonas aeruginosa y Staphylococcus aureus.

En la mayor parte de los estudios realizados en pacientes sin inmunodeficiencia el agente causal más común fue Staphylococcus aureus con porcentajes de manifestación de 15 a 45\%. ${ }^{3-5,10}$ En este estudio, Staphylococcus aureus se aisló en cuatro muestras (17\%); el porcentaje fue similar en pacientes con y sin inmunodeficiencia común variable.

Staphylococcus epidermidis es un colonizador de la cavidad nasal en personas sanas ${ }^{11}$ y Ozcan y colaboradores $^{12}$ concluyeron que no debe incluirse como microorganismo causal de rinosinusitis crónica. En nuestro estudio representó 10 muestras (42\%).

Staphylococcus haemolyticus no se aisló en ningún otro estudio revisado; esto se debe a que este microorganismo afecta con mayor frecuencia a pacientes con inmunodepresión. En nuestro estudio representó una muestra (4\%).
Al igual que en otros estudios, en éste no se aislaron microorganismos anaerobios. Las causas de esto pueden incluir: factores técnicos como contaminación de la muestra, inadecuada técnica de sembrado, así como un medio de cultivo no adecuado para anaerobios estrictos que podrían haber disminuido el número de anaerobios estrictos aislados en nuestro estudio y el tratamiento con corticoesteroides nasales que se administra a los pacientes, que aumenta el drenaje de material purulento y permite una mejor oxigenación de los senos paranasales disminuyendo el crecimiento de anaerobios. ${ }^{2,5,7-8,13}$

También se estudió la incidencia de hongos en nuestros pacientes con rinosinusitis crónica e inmunodeficiencia común variable. Dos muestras tuvieron desarrollo de Candida albicans ( $8 \%$ ) y una muestra de Aspergillus fumigatus (4\%), cifras menores a las reportadas por Araujo $^{4}$ y Panduranga, ${ }^{5}$ pero mayores a lo reportado por Mantovani ${ }^{2}$ y Nigro. $^{14}$

\section{CONCLUSIÓN}

Los microorganismos asociados con rinosinusitis crónica en pacientes adultos con inmunodeficiencia común variable más comunes son: Moraxella catarrhalis y Staphylococcus aureus 
y las bacterias gramnegativas Sphingomonas paucimobilis y Citrobacter koseri. Los agentes fúngicos aislados fueron Candida albicans y Aspergillus fumigatus.

Esto sugiere que a todos nuestros pacientes con diagnóstico de rinosinusitis crónica e inmunodeficiencia común variable debemos tomar cultivo de secreción del meato medio vía endoscópica, para iniciar el tratamiento adecuado principalmente contra Staphylococcus aureus y Moraxella catarrhalis.

De acuerdo con los microorganismos aislados se recomienda iniciar tratamiento con doxiciclina 100 mg vía oral cada 24 horas durante 14 días y, en caso de aislamiento de hongos, se sugiere realizar cirugía funcional endoscópica de nariz y senos paranasales.

\section{REFERENCIAS}

1. Angulo-Pérez G, Vivar-Acevedo E. Prevalencia, localización y severidad tomográfica de rinosinusitis crónica en pacientes adultos con inmunodeficiencia común variable. Revista Alergia México 2015;62:15-21.

2. Mantovani K, Maxillary sinuses microbiology from patients with chronic rhinosinusitis. Braz J Otorhinolaryngol 2010;76:548-551.

3. Majd Z, et al. The microbiology of chronic rhinosinusitis prior to functional endoscopic sinus surgery. Pharmaceutical sciences 2014;20:170.
4. Araujo E. Microbiology of middle meatus in chronic rhinosinusitis. Rev Bras Otorrinolaringol 2007;73:549-555.

5. Panduranga K. Microbiological analysis of paranasal sinuses in chronic sinusitis- A south Indian coastal study. Egyptian J Ear, Nose, Throat Allied Sci 2013;14:185-189.

6. Busaba NY, Siegel NS, Salman SD. Microbiology of chronic ethmoid sinusitis. Am J Otolaryngol 2004;25:379-384.

7. Jiang RS, Hsu CY, Lin JF. Comparison of the bacteriologies between the ethmoid and maxillary sinuses in chronic paranasal sinusitis. J Otolaryngol Soc Roc 1993;28:308-317.

8. Jiang RS, Lin JF, Hsu CY. Correlation between bacteriology of the middle meatus and ethmoid sinus in chronic sinusitis. J Laryngol Otol 2002;116:443-446.

9. Hsueh PR, et al. Nosocomial infections caused by Sphingomonas paucimobilis: clinical features and microbiologic characteristics. Clin Infect Dis 1998;26:676-681.

10. Daza Hernández A. Identificación de Citrobacter koseri como nuevo patógeno en pacientes con rinitis crónica. An Orl Mex 2014;59:1-10.

11. Agnieszka M. Peculiar aspects of rhinosinusitis. En: Microbiology aspects of rhinosinusitis. Disponible en: www. intechopen.com

12. Ozcan M, Unal A, Aksaray S, Yalcin F, Akdeniz T. Correlation of middle meatus and ethmoid sinus microbiology in patients with chronic sinusitis. Rhinology 2002;40:24-27.

13. Brook I, Frazier EH. Correlation between microbiology and previous sinus surgery in patients with chronic maxillary sinusitis. Ann Otol Laryngol 2001;110:148-151.

14. Nigro JFA. Microbiologia dos seios maxilares e etmoidal em pacientes com rinossinusite crónica submetidos à cirurgia funcional endoscópica dos seios paranasais. Braz J Otorhinolaryngol 2006;72:217-222. 